\title{
FORTIFICACIONES MUSULMANAS EN JOLÓ: RESISTENCIA, ADAPTACIÓNY REINTERPRETACIÓN DE LA GUERRA MODERNA OCCIDENTAL
}

\author{
Pedro Luengo \\ Universidad de Sevilla \\ King's College London
}

Resumen: Los estudios sobre fortificaciones no europeas en el sudeste asiático deben incorporarse a la valoración global de este fenómeno en el siglo XVIII. En un momento como éste, en el que los enfrentamientos entre potencias europeas y los sultanatos malayos se sucedían en el tiempo, es posible conocer las características de las defensas islámicas gracias a las memorias de los atacantes europeos. En este trabajo se plantea una primera aproximación a varios casos gracias a fuentes eminentemente españolas, focalizando el interés en los problemas de transferencia tecnológica y en los responsables de este flujo de información. Cualquiera de estas vías supone un enfoque aplicable a otros territorios con contextos similares como son el Mediterráneo o el subcontinente indio.

Palabras clave: Fortificaciones, diálogo cultural, in-betweener, estudios bélicos, conflictos bélicos en el siglo XVIII.

Abstract: The academic approach to no-Europeans fortifications in Southeast Asia has to be included as part of the global evaluation of this phenomenon during the eighteenth century. In such moment, when the conflicts among the European powers and the local sultanates were common, it is possible to know the characteristics of the Islamic defences thanks to the reports by the Western attackers. This work aims to make a first approach to some specific settlements mainly from Spanish sources, focusing its interest on the technological transfer problem and on those responsible of this information exchange. Any of these options can be used as useful approaches for other territories with similar historical contexts such as the Mediterranean or the Indian subcontinent.

Keywords: Fortification, Cultural Dialogue, in-betweener, War Studies, $18^{\text {th }}$ century Warfare.

\section{Introducción}

El estudio de la historia militar en general y de la ingeniería militar en 
particular, han adolecido de un escaso interés por los fenómenos históricos no occidentales ${ }^{1}$. Esto ha sido así incluso en los estudios sobre los siglos XVII y XVIII, cuando los contactos con otras tradiciones bélicas fueron habituales ${ }^{2}$. Sólo cuando fueron las potencias europeas las que se enfrentaron a ejércitos no occidentales, estos han tomado un cierto protagonismo. Siendo esto así para la historia bélica, parece no haber resultado un tema relevante para los estudiosos de la ingeniería militar. Apenas las fortificaciones de la India, y algunas significativas del Mediterráneo han recibido su merecido interés. Para el caso concreto de Filipinas, existen estudios sobre sus fortalezas pero exclusivos de las conservadas, todas ellas levantadas por los españoles ${ }^{3}$."Aun así, resultan necesarias aproximaciones más complejas que comparen las propuestas occidentales y orientales en una misma zona, así como su efectividad defensiva, para más tarde trasladar la comparación a otras áreas similares.

En este contexto, el objetivo de este artículo es plantear una respuesta inicial a cómo afrontaron los asentamientos islámicos del sudeste asiático el desarrollo de la guerra moderna occidental durante el siglo XVIII. Este problema afecta por igual al ámbito mediterráneo, a las posiciones africanas, y a las asiáticas. En esta ocasión serán las posiciones del oeste de Filipinas las analizadas. Aunque los conflictos allí desarrollados no tuvieron la magnitud

${ }^{1}$ Algunos autores anteriores han trabajado para evitar seis líneas de investigación habituales en los estudios bélicos: a) eurocentrismo, b) “maquinización”, c) interés en las “potencias principales”, d) separación de los conflictos navales de los terrestres, e) interés en los conflictos estado vs estado y no en el uso interno de la fuerza, y f) falta de interés en los “órdenes" políticas. Este trabajo pretende ofrecer nuevos datos contra al menos tres de estos puntos. BLACK, Jeremy, Rethinking Military History, London, Routledge, 2004, p. IX. Para el caso concreto que se trata cabe destacar un estudio precedente que también está planteado desde estas directrices LEE JUNKER, Laura, Raiding, Trading, and Feasting: The Political Economy of Philippine Chiefdoms, Honolulu, University of Hawai'i Press, 1999.

${ }^{2}$ BLACK, Jeremy, War in the Eighteenth-century World, Hampshire, Macmillan, 2013.

${ }^{3}$ Cabe destacar los estudios de JAVELLANA, René, Fortress of Empire: Spanish Colonial Fortifications of the Philippines, 1565-1898, Manila, Bookmark, 1997; y más recientemente JAVELLANA, René, "Guarding the Western Frontier: Spanish Colonial Fortifications as a Cultural Route", en GALVÁN, Javier (ed.), Endangered. Fil-Hispanic Architecture, Manila, Instituto Cervantes, 2002, pp. 66-81. Para el caso de Manila, véase LUENGO, Pedro, Manila, plaza fuerte. Ingenieros militares en Manila, 1764 1788, Madrid, CSIC, 2013. 
que los del Mediterráneo o los de India, las descripciones e incluso representaciones gráficas se han conservado ampliamente. Para abordar este problema se analizará una selección de informes sobre ataques europeos a algunas de estas posiciones. A través de estos textos será posible reconstruir no sólo las características de las fortificaciones, sino su efectividad frente a las técnicas occidentales. Una vez identificadas las características de algunas de ellas para el siglo XVIII, se evaluará el grado de diálogo cultural referente a las técnicas occidentales, las islámicas y las locales.

\section{Las fortificaciones del sultanato de Joló antes de la llegada española}

Como han apuntado diferentes estudios arqueológicos, los restos de fortificaciones en el archipiélago filipino han sido datados ya en el siglo XII, aunque los más significativos pertenecen a los siglos XV y XVI ${ }^{4}$. Para este trabajo tienen especial interés los resultados de las excavaciones en Tanjay (Negros Oriental). Aunque los restos arqueológicos del asentamiento se datan en el primer milenio a. C., los restos de fortificaciones se consideran del siglo XV. Así se ha identificado un foso de más de dos metros de ancho con empalizada en el frente costero. A esto habría que añadir otros restos de elementos defensivos en piedra de coral, tierra apisonada y madera junto a residencias de dirigentes joloanos. El modelo de empalizada y foso con agua se extenderá por la zona creando el modelo conocido en tagalo como kuta, en referencia a la empalizada, y bangbang para el foso ${ }^{5}$. Otras soluciones, consideradas por el momento como locales, son las torres de Bicol, conocidas como bantara, o las torres vigías de Zamboanga levantadas en bambú [1].

Para comprender mejor las características de estas empalizadas puede tomarse una descripción más tardía, realizada por Keppel en 1848. Se levan-

\footnotetext{
${ }^{4}$ LEE JUNKER, Laura, Raiding, Trading..., pp. 355-359.

${ }^{5}$ El término parece tener un origen sánscrito, ya que kuta, kotta y kota son sinónimos, significando fuerte. Es significativo que esta pequeña variación entre los tres términos también se mantiene en varias fuentes documentales de Filipinas. Además de en tagalo también se utilizó en javanés antiguo por lo que debería buscarse un modelo común para las fortificaciones del sur y sudeste asiático del siglo XV y XVI, que siguiera en uso con la llegada de los europeos.
} 
taba una doble línea de troncos clavados directamente en tierra separada por un metro y medio. El espacio intermedio se completaba con piedras de gran formato y tierra. La altura del muro alcanzaba los cuatro metros y medio contando con troneras para piezas de artillería. Este modelo de empalizada es muy diferente al que se mantenía en uso por la ingeniería militar occidental, lo que ha podido llevar a errores de interpretación de los textos. Para la teoría militar española del momento una empalizada era un muro de troncos de madera con una altura de unos tres metros de alto que servían como primer parapeto antes de la muralla ${ }^{6}$. No se trataba de una muralla en sí, ya que de hecho algunas se usaban en posiciones oblicuas. Lo que construían los sultanatos asiáticos parecen ser verdaderas murallas de piedra y arena consolidadas por la empalizada en madera. Sólo así sería posible realizar troneras donde colocar la artillería. Este sistema no era considerado una fortificación por los europeos llegando a generar un curioso pasaje entre la expedición de Dalrymple a Joló y la población local. Tras comprometerse a no levantar una fortificación, los británicos acamparon en las afueras, levantando una empalizada alrededor de las tiendas. Los joloanos lo consideraron un primer incumplimiento del tratado que fue explicado como una mala interpretación de lo que era un fuerte ${ }^{7}$.

\section{Descripciones de las fortificaciones por fuentes europeas}

Este modelo de fortificaciones encaja con lo expuesto con más detalle por los españoles. Entre múltiples asentamientos descritos se han seleccionado cuatro, principalmente por la información gráfica disponible.

\section{Joló}

El año 1731, como parte de los planes políticos del gobernador de Filipinas Valdés y Tamón, se generó una interesante documentación sobre varios enclaves [2] (Doc. 1 y 2) ${ }^{8}$. Una expedición salida de Manila el 4 de

${ }^{6}$ ZEPEDAY ANDRADA, Alonso, Epitome de la fortificación moderna, Bruselas, Francisco Foppens, 1669 , p. 127.

${ }^{7}$ FRY, Howard T., Alexander Dalrymple and the Expansion of British Trade, Londres-Nueva York, Routledge, 2013, p. 145.

${ }^{8}$ BARRIO MUÑOZ, José Ángel del, Vientos de reforma ilustrada en Filipinas. El gobernador FernandoValdés Tamón. (1729-1739), Sevilla, CSIC, 2012, p. 72. 
marzo de 1731 se dirigió a Joló, de donde saldría repelida y de donde se conserva una interesante descripción y documentación gráfica. Posteriormente se pasó a Talobo, y de ahí a Bual, cuya fortaleza también queda descrita en el volumen. De ahí la expedición pasaría a la isla de Capual, camino de Zamboanga. Resulta claro que la expedición recorrería el norte de la isla de Joló de oeste a este desde la capital joloana hasta Capual, por lo que los otros dos enclaves deben quedar en medio.

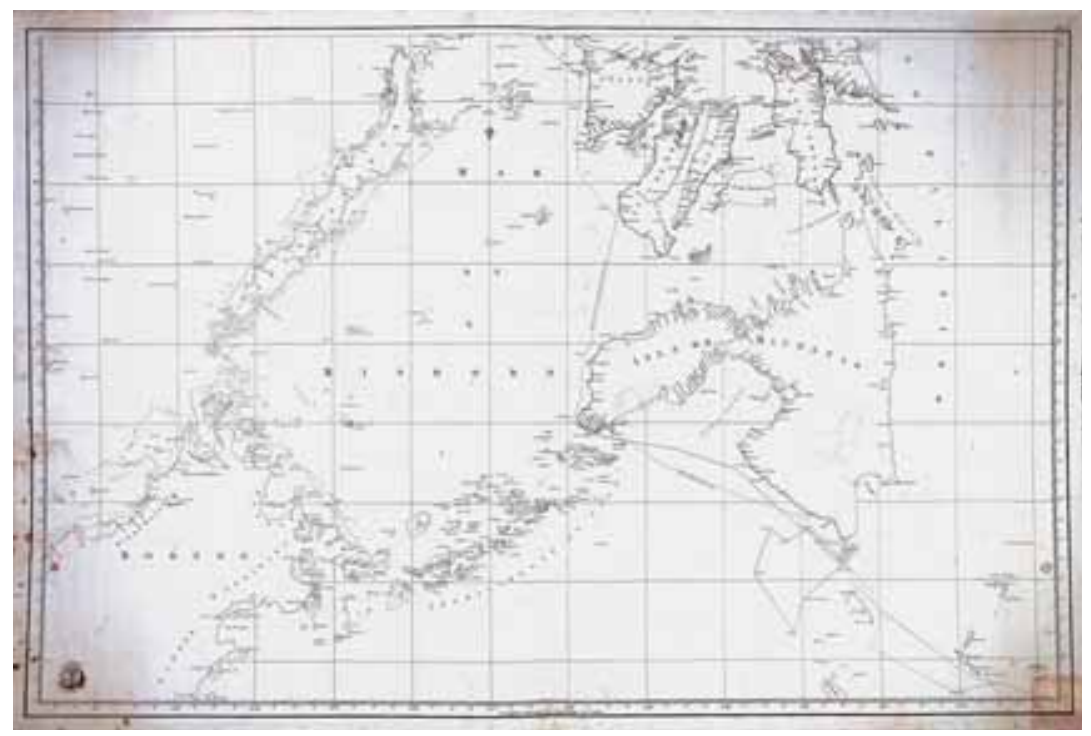

Fig. 1. Mapa del Mar de Mindoro. Archivo General de Indias (AGI), MP-Filipinas, 199.

La descripción de Joló, que puede ser complementada con lo expuesto por Keppel en 1848, es muy significativa, tanto por la descripción gráfica como por la escrita. El dibujo muestra cómo las defensas estaban pensadas de una forma muy diferente al sistema habitual en occidente [2]. En primer lugar, no existía un recinto amurallado pleno alrededor del caserío, sino que sólo el frente de marina quedaba protegido. Está claro que no se esperaba un ataque por tierra o de tipo anfibio, fórmula cada vez más común en el siglo $\mathrm{XVIII}^{9}$. Ciertamente hasta ese momento los ataques navales habían sido los

\footnotetext{
${ }^{9}$ BLACK, Jeremy, War in..., p. 166
} 
habituales de las tropas europeas. Sólo dos recintos se encontraban plenamente amurallado: la "fuerza del rey", identificado con el número 4; y una antigua estructura circular española, identificada con el número 9. De forma significativa, esta última construcción no contó durante el ataque con artillería, ni parece que tuviera un papel específico en la defensa.

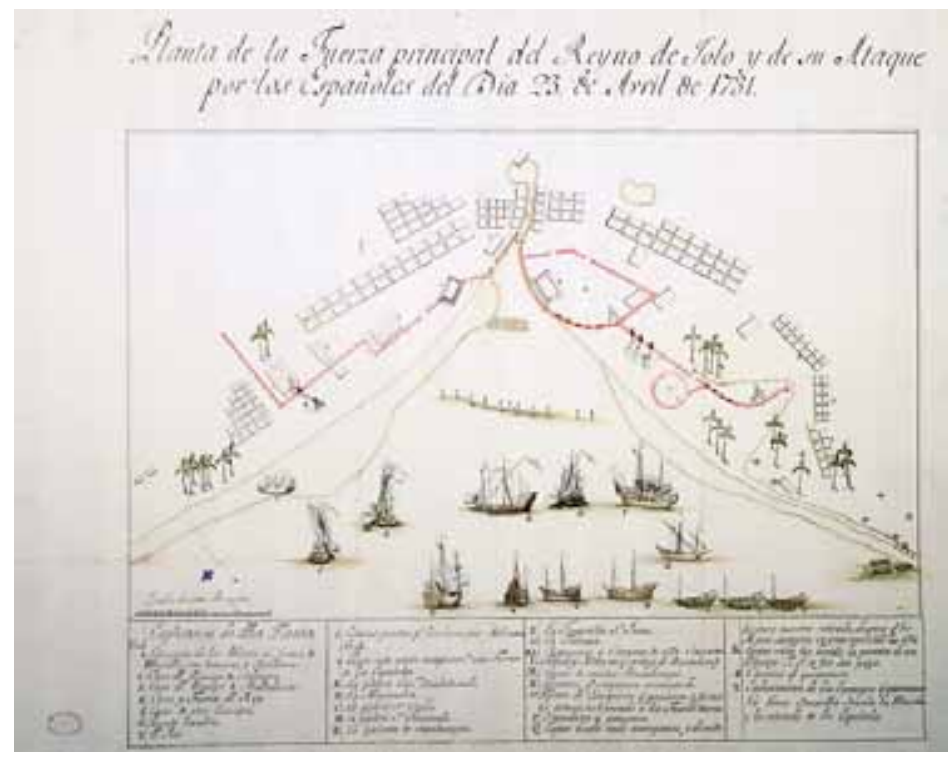

Fig. 2. Planta de la fuerza principal del reino de Joló y de su ataque por los españoles del 23 de abril de 1731. AGI, MP-FILIPINAS, 273.

El perímetro de empalizada tiene un diseño lineal que no busca imitar la forma de los bastiones, o adaptar los modelos geométricos, considerados como los más recomendables para la defensa de una plaza. Se renuncia a imitar cualquier diseño occidental, además de optar por un sistema constructivo, el de las estacadas, diferente al elegido por los enclaves gobernados por potencias occidentales. La ubicación de la artillería tampoco se hace con un orden claro, ya que en el sector sur (derecha de la representación) existen doce troneras, aunque parece que sólo tres contaban con cañones. En cambio, en el sector norte, sólo un cañón y una única tronera parecían sostener ese flanco. Las puertas también requieren análisis. La estacada se interrumpía al menos en cinco puntos. En el sector sur, existía un acceso detrás de la antigua estructura española bien protegida, pero hasta donde llegaron los españoles ${ }^{10}$. Dos accesos, sin protección especial 
pero orientados hacia la población, daban acceso a la fuerza del rey. Otro acceso permitía pasar desde la desembocadura del río a la población, mientras que el último se ubicaba más hacia el norte. En ninguno de los casos se muestra que existiera un control específico de estos puntos. La defensa de la capital se basa en una primera línea de contención formada por una estacada clavada en el lecho marino a la entrada de la desembocadura, flanqueada por artillería en ambos lados como se ha comentado. Una vez pasado estos obstáculos, que los españoles debieron intentar a partir de un ataque anfibio a la estructura circular, el segundo perímetro defensivo sería el de la fuerza real. Aunque el frente marino está protegido por troneras, parece que el lado interior no está preparado de la misma forma, por lo que sería más fácil hacer un asalto desde allí. A pesar de todo, la ciudad resistió este ataque. Joló consiguió mantenerse como un centro importante en la zona hasta que en 1761 se convirtió en objetivo de los gobernantes filipinos. Mientras se preparaba el asalto, en 1763 la ciudad fue tomada por los ingleses. Como parte de esta nueva etapa los ingleses construirían un fuerte que en 1775 sería tomado de nuevo por la población local ${ }^{11}$.

\section{Bual}

Además de este caso, se conoce la descripción de la fuerza de Bual por la misma expedición (Doc. 1). Según las fuentes, este enclave estaba mejor fortificado que Joló, por lo que su descripción resulta relevante. En el documento se utiliza el término baluarte y en otros casos fuerza, por lo que podría pensarse en una estructura en empalizada similar a la descrita en la misma época en Cavicunga (Cagayán) o Fort Hollandois (Banda) ${ }^{12}$. Sería empalizadas con plantas triangulares o cuadradas con bastiones en las esquinas ${ }^{13}$. En caso de repetir los esquemas de Cavicunga se trataría de una destacable muestra de transferen-

${ }^{10}$ Parece probable que las tropas estaban intentando tomar la estructura antigua, donde podrían haber trasladado piezas de artillería y dominar las defensas de este flanco.

${ }^{11}$ GARCÍA DE ARBOLEYA, José, Historia del Archipiélago y sultanía de Joló, y noticia de la expedición española, Habana, Soler y Gelada, 1851, p. 28.

${ }^{12}$ Plano del Fuerte del Presidio de Cabicunga. AGI, MP-Libros_Manuscritos, 81, f. 81r. Para más información sobre estos fuertes y sus representaciones en el siglo XVIII véase LUENGO, Pedro, "Relaciones de plazas en Filipinas durante el siglo XVIII", Revista de Indias -en prensa-.

${ }^{13}$ La mayoría de las estructuras en empalizada no reproducían baluartes en las esquinas como muestran otras estructuras cuadrangulares. Plano del Fuerte del Presidio de Catel. AGI, MP- 
cia tecnológica al reproducir las posibilidades de un bastión. De todas formas, parece difícil de admitir plenamente ya que la descripción continúa diciendo de la fortificación que en "todo era artificiosa, pues para tirar abrían sus puertas y después del tiro la volvían a cerrar haciendo lo mismo en el fuego y manejo de los cañones que según hemos visto son tres". Esta renuncia a las troneras u otras soluciones para la artillería resultan sorprendentes si se cuenta con un bastión.

Tandundala y Sibuyón

A mediados de siglo se continuó con los ataques a enclaves musulmanes, existiendo noticias del ataque a Tandundala y a la torre de Sibuyón. A pesar de que se dio orden de informar sobre las particularidades de las defensas, apenas se conoce que contaban con una torre y artillería en los baluartes. Siguiendo con la explicación ofrecida anteriormente, cabe pensar que los perímetros defensivos con empalizadas representados debieron ser complementados en algunos asentamientos con bastiones del mismo material que alojaran artillería. Esto ofrecería un importante avance tecnológico fruto de la constante defensa del enemigo occidental.

\section{Maacim}

El caso de las fortificaciones del río Maacim, en la costa occidental de la isla de Mindoro en 1770 también resultan interesantes. La localización exacta de la zona puede conocerse gracias a un mapa del asalto [3 y 4 $]^{14}$, que lo ubica en la desembocadura del río Mamburao. En primer lugar, se abordarán las defensas de la desembocadura del río Maasim (hoy Mamburao). Aunque la representación es poco detallada puede interpretarse que la población se encontraba fuera del recinto amurallado que correspondería a la fuerza del gobernante

Libros_Manuscritos, 81,f. 72r; Plano del Fuerte del Presidio de Linao, AGI, MP-Libros_Manuscritos, 81, f. 73r. Plano del Fuerte del Presidio de Capinatan. AGI, MP-Libros_Manuscritos, 81, f. 80r. Plano del Fuerte del Presidio de Ajanas. AGI, MP-Libros_Manuscritos, 81, f. 82r.

${ }^{14}$ Según el catálogo del AGI, tanto MP-Filipinas, 61 como 62 proceden de la carta 62 del gobernador Simón de Anda del legajo AGI, Filipinas, 925 (Manila, 4 de enero de 1771). En el citado legajo existen dos cartas con esa fecha. La primera en el resumen indica "acompaña y recomienda la instancia de Juan Blanco de Sotomayor para que se le confiera el empleo de coronel de aquellas milicias y expone los motivos para remover al marqués de Montecastro". En la documentación no se cita el ataque a Maacim. En la segunda, "que a don Juan Migone y Casamara que pasa a aquellas islas a continuar su mérito de cadete, le tenga presente en las propuestas de oficiales con respeto a su aplicación”. Igualmente, no se trata el ataque. 
local. Este espacio tenía un perfil octogonal, en el que dos de sus lados quedaban libres para servir de accesos. Una de las entradas quedaría protegida por la propia población. De esta forma se protegía de un ataque naval, terrestre o anfibio. La estructura serviría para defender el asentamiento, pero dejaba desprotegido el curso del río por el que subirían los españoles.
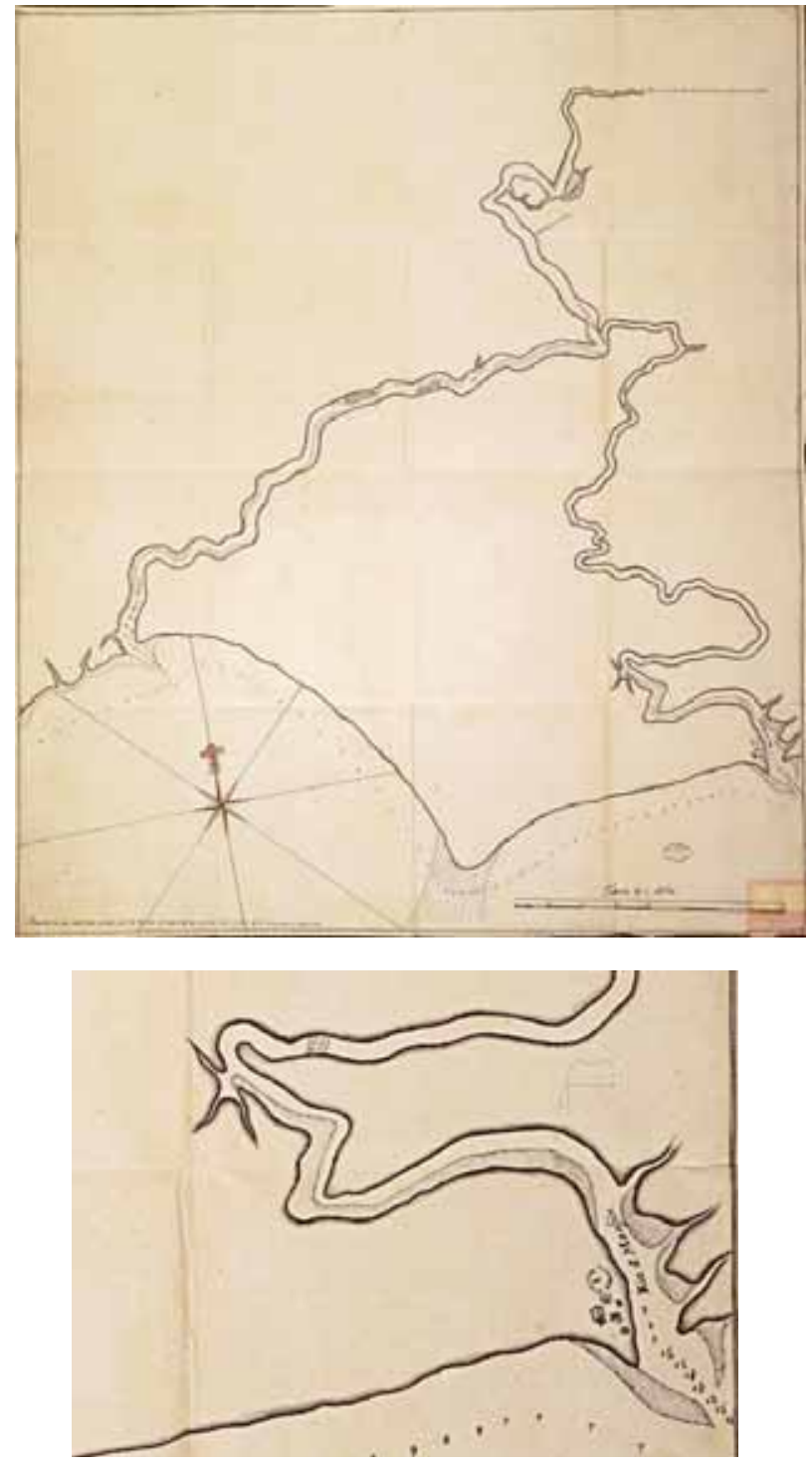

Fig. 3 y 4.Vista general y detalle del Plano de situación y fortificación de la embocadura del Río Maassin.AGI, MP-FILIPINAS, 62. 
En segundo lugar, cabe estudiar las defensas ubicadas río arriba [5]. En este caso el perímetro también es cerrado, con un perfil irregular, marcando una clara diferencia con las propuestas más antiguas de Joló. En este caso, el carácter irregular parece necesario por la orografía del terreno, pudiendo considerarse incluso un intento de mejorar el control sobre algunos puntos, incluyendo fórmulas similares a pequeños bastiones como en el lado este (lado derecho en la representación). El número de troneras alcanza catorce, repartidas de forma regular entre los diferentes frentes con excepción del lado sur (parte baja de la representación) ${ }^{15}$. Parece ser que el número de acceso se había reducido a uno, que sería posible controlarse desde el frente del río. Cabe señalar que no existían torres ni estructuras de apoyo a lo largo del río que hubieran podido ayudar a la defensa. Podría plantearse que existió una asimilación de conceptos occidentales a lo largo del siglo XVIII.

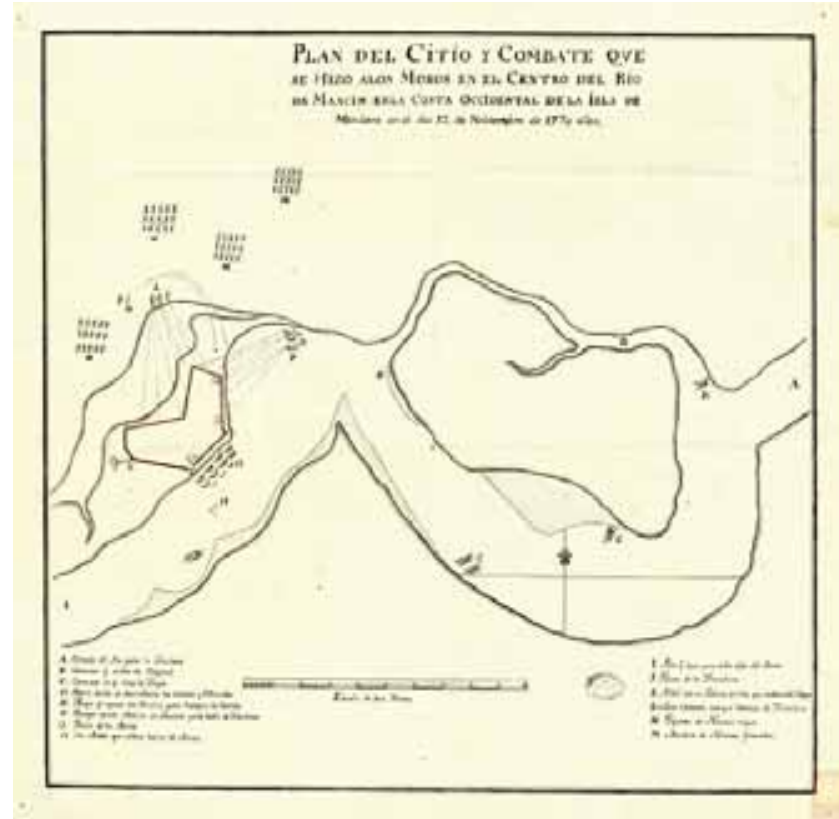

Fig. 5. Ataque en el río Maacim (hoy Mamburao) en la costa occidental de Mindoro. AGI, MP-FILIPINAS, 61.

${ }^{15}$ Las orientaciones de los dos mapas no concuerdan. Ambas representaciones tienen el norte en la parte superior, mientras que los perfiles están colocados invertidos. Parece claro que es el MPFilipinas, 61 el que tiene orientación sur, habiendo dibujado la flecha equivocadamente. Para evitar más confusión, se ha mantenido el error en la descripción. 


\section{Transferencia tecnológica y in-betweeners}

A partir de estas descripciones puede decirse que las fortificaciones no respondían a adaptaciones locales de modelos externos, ya fueran occidentales, chinos o indios. En el primer caso, resulta difícil considerar que la organización más básica de una fortificación abaluartada se siguiera aquí con empalizadas. Algo similar puede decirse del ámbito indio ${ }^{16}$. Aunque las fortificaciones indias se siguieron construyendo sin baluartes, las torres semicirculares en perímetros amurallados, los fosos, las murallas barbacanas o las entradas en recodo se mantuvieron en uso. Sobre China se puede indicar algo similar. Desde época anterior a la llegada de la dinastía Qing, las murallas de piedra con torres cuadradas y grandes puertas bien protegidas fueron habituales en muchas ciudades chinas. Incluso, aunque de forma tardía y con interesantes adaptaciones, cabe destacar en este sentido la fortaleza de Hwaseong (Corea del Sur) construida por JeongYak-yong entre 1794 y 1796. Rechazados los modelos de las grandes potencias bélicas del entorno, cabe decir que los joloanos mantuvieron un modelo que resultaría arcaico y deficiente para las potencias europeas y quizás también para los piratas chinos.

Una posible explicación para entender la repetida elección del sistema de empalizadas por parte de los gobernantes islámicos puede deberse a la dificultad de contar con personal especializado en el diseño de otro tipo de defensas. En primer lugar cabe señalar que las internadas de estos moros en tierras fortificadas por españoles fueron continuas tanto en la zona norte de la isla de Paragua, como incluso en Manila ${ }^{17}$. Pero mucho más importante es subrayar que hubo militares que trabajaron en la capital y finalmente pasaron al lado enemigo. Un caso anecdótico pero significativo es el de Cayetano Balcazar y Bernardo Betancur (Doc. 1). El primero, identificado como moro en el documento, había residido en Manila y conocía de esta etapa al soldado Betancur, hasta el punto de saludarle tras el cese del fuego. Como este caso conocido, es probable que la mano de obra usada por los ingenieros militares en Manila fuera local, siendo previsible que algunos pudieran huir al sur del archipiélago, llevando consigo unos mínimos rudimentos de fortificación.

\footnotetext{
${ }^{16}$ DELOCHE, Jean, Studies on Fortification in India, Pondichéry, Institut Français de Pondichéry, 2007.

${ }^{17}$ Expedientes sobre defensa (1771-1773). AGI, FILIPINAS, 925.
} 
Algo similar ocurre con aquellos que participaran de la construcción de fuertes en Salay, Cugman, Mambahao, Lubungan o Iponam, que incorporaban baluartes tanto en piedra como en madera bajo supervisión de los misione$\operatorname{ros}^{18}$. Estos casos pueden identificarse como inbetweeners, es decir, aquellos que conocen y participan de varias tradiciones culturales normalmente en diálogo. A pesar de estos ejemplos, las diferentes descripciones de Joló a lo largo del siglo XVIII, e incluso del siglo XIX, apuntan a que este conocimiento no se aplicó, probablemente, por decisión de los gobernantes locales.

\section{Efectividad ante ataques europeos}

Esta divergencia frente a los modelos habituales podría considerarse un localismo en caso de tratarse de otro tipo de manifestación cultural, pero los joloanos debían protegerse con estas defensas por lo que la renuncia a otras fórmulas más eficientes debe evaluarse de forma diferente. Antes es necesario conocer cómo se comportaron estas estructuras, teóricamente deficientes según la teoría europea del momento, ante los ataques occidentales. En primer lugar, cabe señalar que pocas de estas posiciones terminaron cayendo definitivamente en manos europeas hasta fecha más tardía. Apenas los casos de Iponan y Linamon han sido descritos con cierto detenimiento:

"bajasen los monteses al socorro capitaneados del maestre de campo Amigos y del sargento mayor Dagojan quienes viendo la dificultad de socorrer a Iponan, porque la tenían los moros sit[i]ada con trincheras y nueve baluartes determinaron hacer el último esfuerzo de valor para liberar a Iponan"19.

"partió el padre con la armada para Linamon donde estaban hechos fuertes los moros con sus estacadas pero la armada los obligó a puros lantacaso a que desalojaran aquel puerto perfeccionando la acción con el desembarque de los nuestros que destru//yeron las fortificaciones de los moros" ${ }^{20}$.

Por tanto, los sistemas defensivos fueron en alguna medida exitosos. Su comportamiento en la defensa queda bien descrito en los informes. Des-

\footnotetext{
${ }^{18}$ WARREN, James Francis, The Sulu Zone, 1768-1898. The Dynamics of External Trade, Slavery and Ethnicity in the Transformation of a Southeast-Asian Maritime State, Singapur, NUS Press, 2007, p. 293.

${ }^{19}$ Sitio de Iponan (¿Cagayán de Oro?) por los moros. AGI, FILIPINAS, 709, f. 33v.

${ }^{20}$ Fuerte de Linamon (Lanao del Norte). AGI, FILIPINAS, 709, ff. 40r-40v.
} 
de las empalizadas se mantenía un ataque continuado con artillería, lantacas y cerbatanas. Esto no impedía que las tropas españolas se acercaran al muro, por lo que puntualmente salían las tropas locales a defenderse con gran número de soldados. Este tipo de asaltos, según las fuentes españolas, solían finalizar con un alto número de pérdidas para los defensores. Hasta aquí, resultaría fácil para los atacantes hacerse con la posición, pero en varios casos las circunstancias climatológicas obligaron a la retirada. En Bual se trató de un fuerte aguacero (Doc. 1), mientras que en otra factoría de Borneo fue un vendaval (Doc. 2). En otros casos, parece que un ataque masivo local obligaba a retirar las tropas de tierra, y a dejar el asalto español en una acción meramente intimidatoria. Esto puede llevar a pensar a que los gobernantes islámicos intentaban defenderse de un ataque en el que el paso del tiempo jugaba en su favor. Los cambios de vientos y mareas o la llegada de aguaceros suponían un importante revés para las tropas occidentales, que por otro lado, parecían mantener una intención más intimidatoria que conquistadora.

\section{Conclusiones}

A partir de los aspectos presentados se pueden recopilar algunas conclusiones. En primer lugar, que los gobernantes del sultanato de Joló apostaron por un modelo propio de sistema defensivo, sin nexos claros con otras potencias militares del momento en la zona. Esta opción, que es previa a la llegada europea, se mantuvo durante el siglo XVIII sin cambios sustanciales. Estos enclaves sufrieron numerosos ataques pero con muchas dificultades y gracias a otros factores, consiguieron no ser conquistados. Esto les permitió mantener la confianza en un sistema barato y rápido de construir frente a otros modelos que debían conocer por personal previamente al servicio europeo. Por tanto, parece claro que el carácter pragmático, incluso cuando su efectividad fuera muy limitada, se impuso a la actualización tecnológica que proponía el diálogo cultural con las potencias occidentales. Tal debió ser el éxito y coste de estos fuertes que incluso los europeos llegaron a copiarlos, planteando un sugerente fenómeno de diálogo cultural:

“me mantuve en dicha ensenada [puerto de Mobo] para amonestar a los naturales para demoler la cota y estacada y fabricar la que está mandada 
levantar en el sitio que señaló el general don Tomás de Iturralde con acuerdo del reverendo padre ministro lo que no ejecuté aún habiéndoles concedido anteriormente el término de tres meses para formar dicha estacada" ${ }^{21}$.

Sólo a finales del siglo XVIII se observa una apuesta de los sultanatos por conseguir aliados europeos, y en estos casos se pasa de la prohibición a levantar fuertes a la petición de hacerlo ${ }^{22}$. Este fenómeno de renuncia de transferencia, que se puede observar en otros puntos del sudeste asiático como China o Japón hasta muy entrado el siglo XIX, resulta de un gran interés y podrá ser comparado con estos casos cuando se aborden monográficamente.

\section{Anexo documental}

Documento 1. Ataque a la fuerza de Bual, Mindanao. AGI, Filipinas, 195, N. 23. 14 de junio de 1731.

f. 11Av. "28. Día veintiocho descu/brimos el pueblo con su baluarte de estaquería y en él arboladas dos banderas rojas por lo que llamé a junta a los jefes de las embarcaciones que me acompañaban y en dicha junta se resolvió el que se le diera batería a dicho fuerte con desembarque de la gente de armas que / /11Br. Se pudiese y con ocho parecer a proa más las embarcaciones a tierra, y no bien dado sordo cuando del dicho fuerte nos tiraron un cañonazo que al gobierno San Carlos le hizo la campana pedazos dejándose sin sentido a la postre que arrimado a la campana había más sin haber le agraviado en nada y entre ocho y nueve se empezó la batería de las dos galeras y la galeota San Juan Bautista con la artillería gruesa, haciendo aún mismo tiempo el desembarque con cuatrocientos hombres de armas, de tal suerte que pusieron espanto el modo con que dicha gente se desembarcó y fue puesta en orden marchando hasta ponerse debajo de la misma estaquería del baluarte // 11Bv. De donde salieron por tres veces a pecho descubierto a pelear nuestra gente y luego la fuerza empezaba a dar carga cerrada a la gente que salía a batalla con la mía y ellos entonces se volvía a retirar, habiendo descubierto reforzado de esta capitana, una emboscada que tenían hecho de más de veinticinco o treinta hombres sin haber quedado de ellos sino como cinco o seis que escaparon por haberse metido en la fuerza según lo ha informado y de dicha fuerza con tal destreza y prontitud a hacer el fuego a nuestra infantería con lantacas y cerbatanas que alrededor tenía de su estaquería la que en todo era artificiosa, pues para tirar abrían sus puertas y

\footnotetext{
${ }^{21}$ Expediente sobre la expedición de Pedro Gaztambide en 1756. AGI, Filipinas, 199, n. 13, FF. 60v-61r.

${ }^{22}$ FRY, Howard T., Alexander Dalrymple... p. 138.
} 
después del tiro la //12Ar. Volvían a cerrar haciendo lo mismo en el fuego y manejo de los cañones que según hemos visto son tres. La falúa viendo las nayaras que hacían con precautela de salir huyendo por una puerta y entrar por otra se fue metiendo tan debajo de la dicha fuerza y estaquería para desde allí con granadas y frascos de pólvora abrazar los que hubiese dentro de dicha fuerza más ellos en todo astuto lo dejaron bien arrimar a su salvoconducto, le tiraron dos tiros con las estacas o pedrero, por lo que dicha falúa se fue haciendo de debajo de la fuerza haciendo fuego con cuatro pedreros por dicha falúa tiene sin embargo de que por varias partes se arrojaron dentro, algunas granadas duró la // 12Av. Batería cerca de cinco horas y por tener encima el aguacero, mandó a pedir la retirada el capitán don Andrés de Palacios, cabo de aquella gente por lo que le respondí obrase en todo como quien tiene la cosa presente por lo que se fueron retirando y un moro llamado Cayetano Balcazar, desde dicha fuerza llamaba con grandes instancias a un soldado compañero amigo suyo, de esta capitana llamado Bernardo Betancur pues él y dicho soldado fueron mucho tiempo compañeros pues vivían y comían en una misma casa en la ciudad de Manila y por no sé qué travesuras fue enviado a Calamianes y de Calamianes se huyó al monte y luego que los joloes pusieron el sitio se unió con // 12Br., ellos, coadyudando a dichos moros, en la invasión y saqueo con otros dos compañeros que no sabemos quienes son, pero estando nuestra gente embarcando se les empezó a caer el aguacero habiendo tenido en este refriega seis muertos y cinco heridos, habiendo quedado en tierra los malogrados cuerpos. Andrés Villavicencio, y Agustín Maclit, forzados de esta capitana siendo este fuerte tan fortificado en armas y gente como Joló y está más fortificado, con nuevas estaquerías, a las tres de la tarde en dicho día nos levamos habiendo primero despachado la taratana / / 12Bv. con los heridos para que los curasen en la enfermería que quedó en compañía de la Almiranta en la Bahía de Joló. Este pueblo y fuerza se llama Bual y el que lo gobierna es Balasan Principal de Mindanao y yerno del Rey viejo de Tanítaní y según dicen algunos lutaos que han estado allí, se halla dicho balasan con más de cuatro mil confederados. [...] f. 13Bv. "nos levamos y con la marea y un poco de viento llegamos antes de las nueva a Joló y dimos fondo y registrado el puerto le he hallado con muchas novedades lo primero por hallar dos banderas más arboladas y por la parte que mira donde hicimos el desembarque hallamos con nueva estaquería desde la playa hasta el manglar y quitado todas las caserías que quemó nuestra gente dejando limpia toda la campaña y por la otra punta que mira en donde está la casa del príncipe Salicaya fueron quitadas las casas y puesta una estaquería mediana, seguida desde su // 14Ar. Casa al monte dejando asimismo limpia la campaña por esta otra parte y este mismo día llegamos cuatro tropas de gente [...] f. 14Ar. Día cuatro, se determinó por registrar el pueblo de Parán por ver si por alguna parte se le podía ha//14Av. Cer algún daño.

\section{Documento 2. Fernando Valdés y Tamón, julio de 1731. AGI, Filipinas, 143, N. 14. S.f. (se incluyen los números de imágenes de la digitalización)}

12. "En una de las instrucciones se le prevenía al general, que a su retirada, reconociese la isla de la Paragua, o Calamianes, y en ella procurase asolar una pequeña fortificación, que en la parte que tienen factoría los burneyes, habían fabricado estos, y distintas naciones para salir a sus correrías, no le fue posible a dicho general cumplir el orden men- 
cionado, porque lo adelantado del tiempo y los vientos vendavales que reinan en aquella parte, no le permitirían navegar sus mares, y fuera exponer las galeras a una ruina”. [AGI, MP-FILIPINAS, 273].

692. "Y hecho el daño que se pudo, no se ha experimentado movimiento alguno en aquellos enemigos los que ya no salen como antes con sus armadillas, antes bien horrorizados del castigo, solo se aplican a fortalecer sus puertos, y ensenadas en el modo que les es posible contentándose con defender sus casas olvidados ya de ofender las ajenas en que estaban tan engreídos por una, u, otra contingencia que lograron en los pueblos de los nuestros que hallaban poco o nada. El gobernador de Zamboanga no ha cesado en hacerles guerra invadiéndoles la Isla de Basilan, que es por su fertilidad el granero de los régulos de Joló, quienes unidos a una isla, con sus sácopes, o principales parece según noticia de algunos cautivos, que han introducido plática de paces.

Documento 3. Ataque español a Tandundala y Sibuyón entre 1750-1751. AGI, Filipinas, 707, N. 1 .

f.2r."dirigiendo su derrota a la ensenada de Joló donde dio fondo a los 26 de junio de dicho año a distancia de una milla de sus fortalezas, y puesto en línea de combate, y reconocido que dos champanes de China estaban fondeados sin bandera en la boca del río que iban tendiendo espías para entrarlos destacó inmediatamente dos lanchas en guerra para sacarlos de debajo de la artillería enemiga cuando comenzaron los joloes desde sus cuatro fuertes con banderas rojas a hacer fuego a las embarcaciones remolcantes, los referidos champanes con artillería de ocho hasta dieciocho de calibre a que correspondiendo nuestra armada se fue continuando el disparo de una y / /2v. otra parte por algún tiempo, entre tanto que mudando los enemigos de bandera para dar lugar al refuerzo de sus trincheras despachó el maestre de campo una carta al príncipe Asim, significándole que el fin de aquella armada era solamente para restituir a la posesión de aquellos dominios a su legítimo señor y recuperar los cautivos a que respondió dicho príncipe que no tenía cautivos que entregar que aguardaban a su rey, para que dispusiese de ellas y que estaban pidiendo a su profeta volviese a su rey a su reino y que como obedientes a su dato, y sultán don Fernando I, no podían tratar de los // 3r. cautivos que los sácopes vasallos de Basilan tendrían escondidos. [...] [Nueva foliación] f. 5r. El veintinueve amanecieron arboladas las tres banderas rojas a la fuerza del rey y torre de Sibuyón, y también la blanca que pusieron ayer en Tandundala, y poco antes de salir el sol, comenzaron a tirar los enemigos, pero con la lentitud que el primer día y algunos cañones menos, que me dio motivo de afirmarme en el pensamiento de que estaban muy maltratados los baluartes, o que iban retirando los cañones. [...] f. 9r. "el primero de julio habiendo amanecido se vio que en Tandundalaga habían quitado la bandera blanca y arbolado otra que parecía amarilla. Al salir el sol mandé dar tres voces viva el Rey y que extendiesen la bandera de popa. Como a las nueve del día viendo que los enemigos no han tirado más que un tiro, y la novedad de la bandera mandé que fuese la lancha en tierra a saber si quería decir algo con la nueva señal y al mismo tiempo hice cesar el fuego. [...] f. 10v. "y registrar y demarcar mejor la fortaleza, el puerto, el río, y sus inmediaciones”. 